\title{
- Toxinologia veterinária on-line
}

\section{- On-line veterinary toxinology}

\author{
AndréLuis Cherubini ${ }^{1}$-CRMV-SP n ${ }^{\circ} 10.656$ \\ Pedro Luís Bicudo ${ }^{2}$ - CRMV-SPn ${ }^{0} 0644$ \\ Rui SeabraFerreiraJunior ${ }^{3}$-CRMV-SP $n^{0} 12.240$ \\ * Benedito Barraviera ${ }^{4}$ - CREMESPn 31.434 \\ 1 Aluno do Curso de Aprimoramento em Animais Peçonhentos \\ da Faculdade de Medicina - UNESP - Botucatu. \\ 2 Professor Assistente Doutor aposentado da Faculdade \\ de Medicina Veterinária e Zootecnia - UNESP - Botucatu. \\ 3 Aluno do Curso de Aprimoramento em Animais \\ Peçonhentos da Faculdade de Medicina- UNESP - Botucatu. \\ 4 Professor Titular do Departamento de Doenças Tropicais \\ e Diagnóstico por Imagem da Faculdade de Medicina - UNESP - Botucatu.
}

* CEVAP - Centro de Estudos de Venenos e Animais Peçonhentos da UNESP Campus de Botucatu - SP http://www.cevap.org.br

\section{RESUMO}

Os autores apresentam de maneira sucinta a importância das publicações sobre Toxinologia para a Medicina Veterinária. Os objetivos principais destas são o de poder auxiliar e contribuir para uma melhor formação dos alunos de Medicina Veterinária do Brasil, além de proporcionar atualização aos profissionais da área. Assim, foi descrito resumidamente o contexto e a importância da Disciplina, bem como, as publicações em papel e CD-ROM pelo CEVAP. Além disso, estão disponíveis na Internet, páginas dedicadas exclusivamente às Emergências Veterinárias, às quais encontram-se inseridas no Centro Virtual de Toxinologia.

Palavras chave: Toxinologia, toxicologia, toxinas, animais peçonhentos.

\section{Introdução}

A toxinologia é a parte da ciência que se dedica ao estudo das toxinas e dos derivados de plantas, de microorganismos (bactérias e fungos) e de animais (serpentes, sapos, peixes, aranhas, escorpiões, abelhas, vespas, formigas, lagartas, animais marinhos, etc...). Tem aspecto multidisciplinar e abrange várias áreas de conhecimento científico, a saber: médica, veterinária, agronômica, zootécnica, farmacêutica, bioquímica e biológica de maneira geral (BARRAVIEIRA, 1998).

Neste contexto o papel do médico veterinário é imprescindível, pois este profissional dedica-se a um setor no qual há especial interesse pelos demais: a produção dos soros heterólogos. Além disso, sua importância é fundamental no atendimento aos animais domésticos molestados por animais peçonhentos.
Entre os animais peçonhentos, isto é, produtores de venenos, podemos dar ênfase às serpentes, que se constituem em quatro gêneros de importância médicoveterinária no nosso País a saber: Bothrops, Crotalus, Micrurus e Lachesis.

Do ponto de vista médico, as serpentes do gênero Bothrops são responsáveis por cerca de 80 a $90 \%$ dos acidentes ofídicos observados no Brasil. As principais espécies desse gênero são as jararacas (Bothrops jararaca) e urutus (Bothrops alternatus), muito comuns nas regiões Centro-Oeste, Sudeste e Sul. No Nordeste predomina a Bothrops erythromelas, cujo nome popular é jararaca-da-seca e no Norte a Bothrops atrox, conhecida também por surucucu. No Centro-Oeste e parte da região Sudeste existe a Bothrops moojeni, (Figura 1) conhecida também por caiçaca. As serpentes do gênero Bothrops causam acidentes na maioria das vezes de leve à moderada gravidade, podendo tornar-se graves quando 
não tratados precoce e adequadamente (BARRAVIEIRA, 1998).

As serpentes do gênero Crotalus (Figura 2) são responsáveis por cerca de 10 a $20 \%$ dos acidentes, dependendo da região do país avaliada. Esses acidentes são mais graves que os causados por Bothrops e podem levar o doente ao óbito. As serpentes do gênero Micrurus habitam todo o país e são denominadas de cobras corais venenosas ou verdadeiras. As do gênero Lachesis são predominantes na região Amazônica e parte da Mata Atlântica e são popularmente conhecidas por surucucu pico-de-jaca. Os acidentes por Micrurus e Lachesis são extremamente raros, porém os primeiros são de extrema gravidade.

Do ponto de vista veterinário, as serpentes do gênero Bothrops e Crotalus são as maiores causadoras de acidentes. Cerca de $90 \%$ deles são causados pelas Bothrops.

Os acidentes com anfíbios têm especial relação com os cães, uma vez que estes atacam e mordem os sapos (Figura 3). O veneno liberado diretamente na boca do agressor acaba intoxicando o animal, podendo evoluir de forma grave chegando até o óbito.

Como se pode perceber, pelos motivos expostos, a Toxinologia é uma Disciplina que tem especial importância, tanto para a área médica quanto veterinária, sobretudo nas regiões tropicais do planeta. Por outro lado, a escassez de livros especializados na área veterinária e a pequena preocupação das Escolas Médico-Veterinárias em abordarem o assunto, tornam o treinamento dos alunos deficitário (BARRAVIEIRA, 1992; BARRAVIEIRA 1996a). Assim, estas Escolas deveriam incluir este assunto definitivamente nos seus currículos, abordando sobretudo o tema com os seus médicos veterinários recémformados, quando os mesmos se encontram em fase de especialização.

Foi baseado nessas dificuldades que a equipe do CEVAP, Centro de Estudos de Venenos e Animais Peçonhentos da UNESP - Campus de Botucatu, decidiu contribuir com o ensino da Toxinologia nas Escolas Médicas e Veterinárias do Brasil.

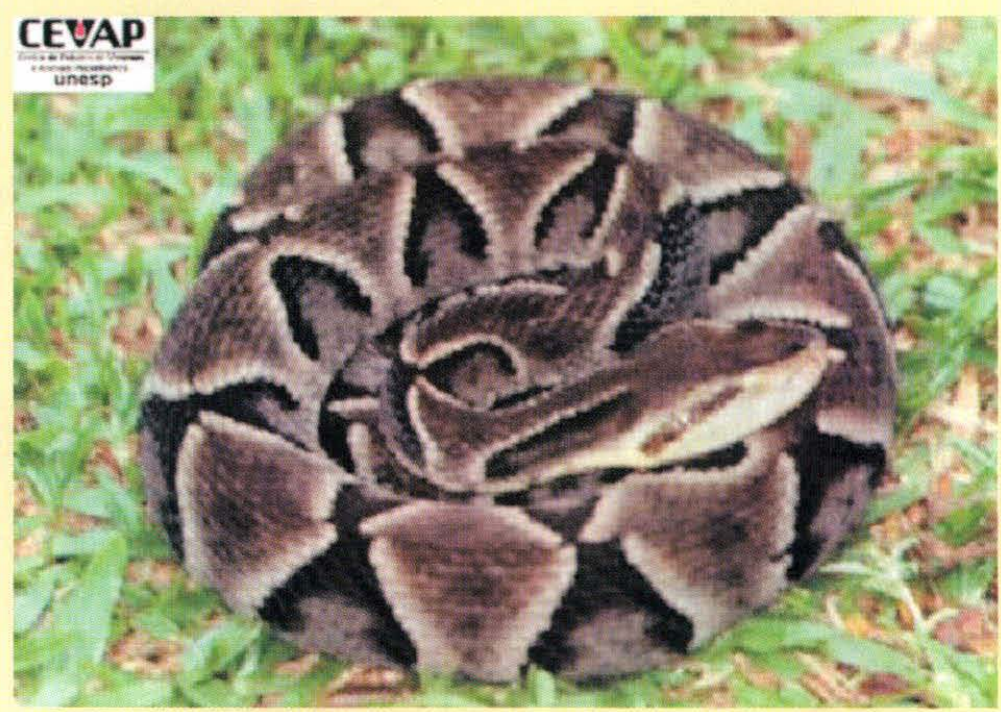

Figura 1: Bothrops moojeni.

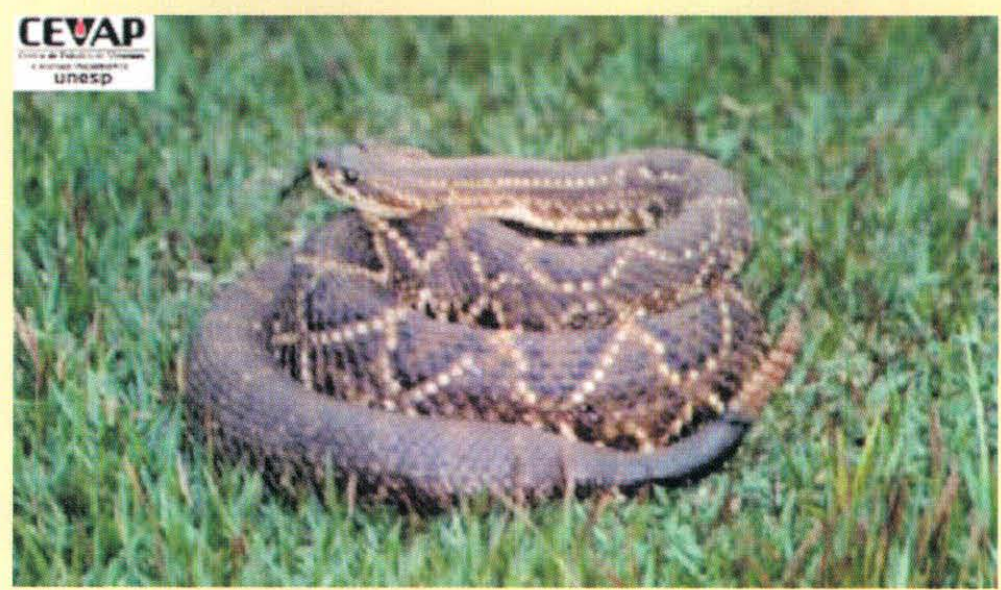

Figura 2: Crotalus durissus terrificus.

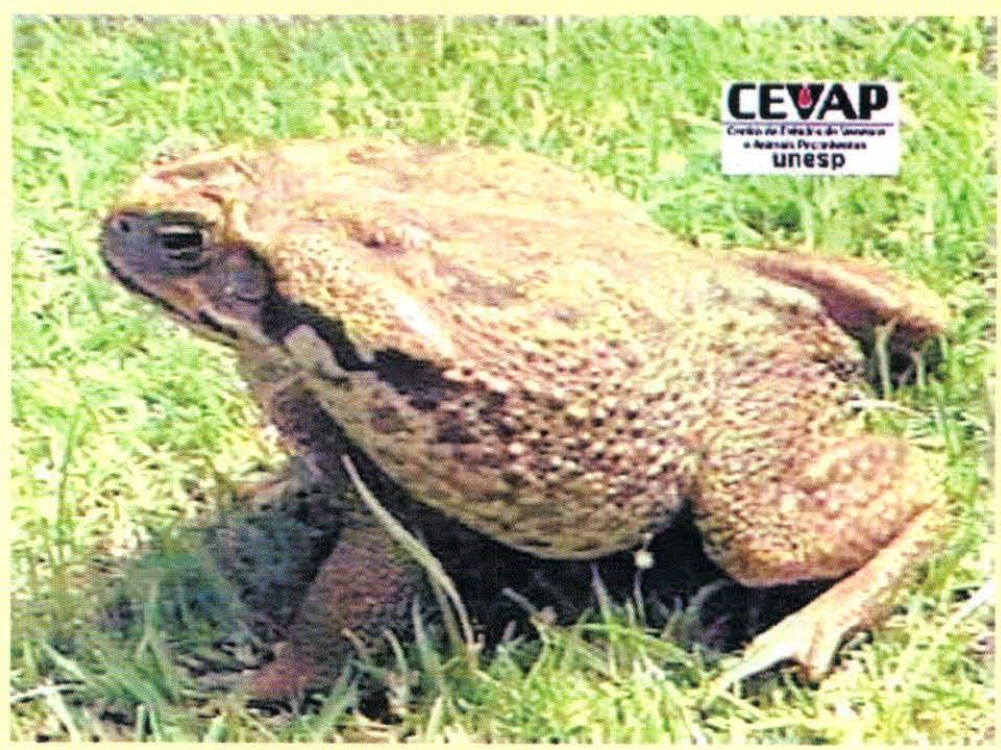

Figura 3: Bufo sp. 


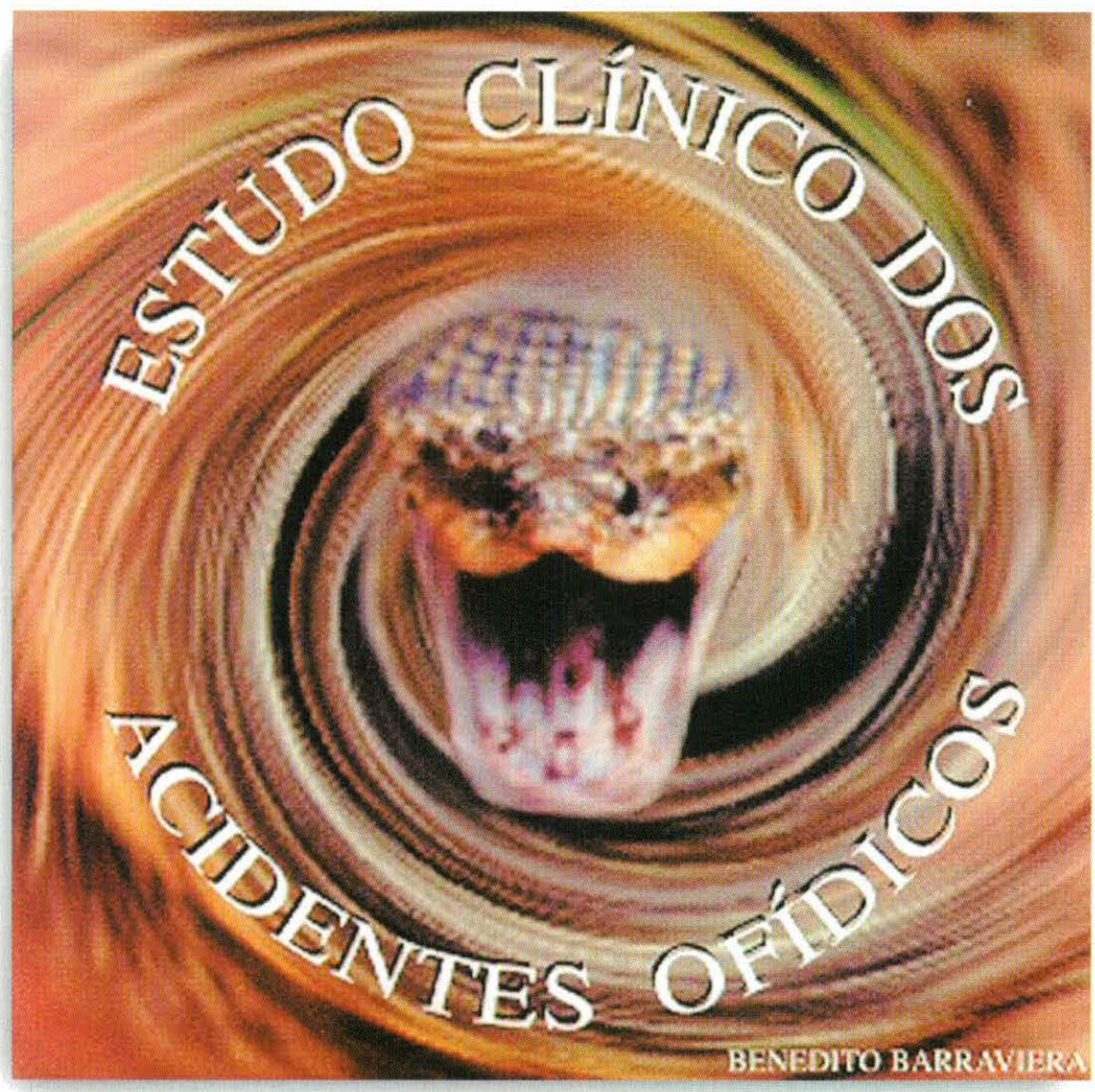

Figura 4: Logocapa/CD. Estudos clínicos dos acidentes ofídicos

Este Centro, criado em 1989, possui uma equipe multidisciplinar, com a finalidade de promover a pesquisa básica, aplicada e tecnológica, o ensino de especialização e pós-graduação e a assistência à comunidade.

Assim em 1994, foi lançado no mercado editorial pela EPUB (http://www.epub.com.br) o livro do CEVAP, intitulado: Venenos animais: uma visão integrada, atualmente Venenos: aspectos clínicos e terapêuticos dos acidentes por animais peçonhentos (BARRAVIEIRA, 1998), escrito por 23 colegas do Centro e 13 colaboradores de outras instituições de pesquisa do Brasil. Esse livro é dedicado aos profissionais das áreas biológica, médica e veterinária.

Em 1995, com o objetivo de dar velocidade às publicações científicas, os pesquisadores da UNESP disponibilizaram a primeira revista eletrônica científica do Brasil. Denominada "The Journal of Venomous Animals and Toxins" (BARRAVIEIRA, 1996a), com periodicidade semestral e uma tiragem de cerca de 1.300 exemplares, é distribuída gratuitamente para todos os sócios da Sociedade Brasileira de Toxinologia e "International Society on Toxonology". Essa publicação é escrita em língua inglesa e distribuída em disquetes para a comunidade científica, tendo sido disponibilizado seus "Abstracts" na Internet em 1996.

No ano seguinte, em virtude do crescimento vertiginoso da Internet (BARRAVIEIRA, 1997) e da carência de informações técnicas nesta área do conhecimento científico, o CEVAP disponibilizou o Centro Virtual de Toxinologia. (http://www.cevap.org.br) . Este Centro, escrito nas línguas inglesa e portuguesa, disponibiliza todos os "Abstracts" da revista eletrônica, além de páginas denominadas Emergências Médicas especialmente dedicadas à classe médica.

Em 1998, o The Journal of Venomous Animals and Toxins foi convidado a participar do projeto SCIELO - uma iniciativa FAPESP/BIREME, cujo objetivo é criar a primeira biblioteca virtual do Brasil e disponibilizar todas as nossas revistas científicas (BARRAVIEIRA, 1997).

Como se pode observar, a Internet veio contribuir com a possibilidade de se construir centros de informações e de consultas virtuais. Essas informações, em decorrência da tecnologia disponível, podem ser alteradas, atualizadas e modificadas a qualquer instante e de qualquer lugar do mundo.

Conhecida como a maior rede mundial de comunicação do século XX, desde a sua chegada, vem mudando o comportamento dos pesquisadores do mundo inteiro. Ela trouxe praticidade, rapidez, alterou o comportamento de seus usuários, demoliu fronteiras e tornou-se uma fonte inesgotável de pesquisa. Além disso, criou o espírito do ensino à distância (BARRAVIEIRA, 1997).

O CEVAP, inserido nesse contexto, disponibilizou em 1999 um CD-ROM intitulado Estudo Clínico dos Acidentes Ofídicos (BARRAVIEIRA, 1999) (Figura 4) (http://www.epub.com.br) e as páginas denominadas Emergências Veterinárias, dentro do Centro Virtual de Toxinologia. (http://www.cevap.org.br).

O CD-ROM contém todas as informações técnicas relacionadas com os acidentes ofídicos no Brasil. É dividido em 8 capítulos: histórico, classificação das principais serpentes brasileiras, epidemiologia, patogenia, quadro clínico, diagnóstico, tratamento e profilaxia. O CDROM roda sem necessidade de instalação em cima da plataforma Windows 95 ou superior. Contém 345 figuras digitalizadas, 100 testes do tipo certo-errado comenta- 
dos, 20 testes de múltipla escolha e com imagens comentados, 70 testes do tipo múltipla escolha comentados, 2 discussões de casos clínicos, 615 botões interativos de navegação, 75 imagens interativas, 2 clipes de vídeo (histórico do CEVAP e os acidentes ofídicos no Brasil), 5 animações, 88 locuções dos principais tópicos, 9 botões principais de navegação, música tema inédita, 12 gráficos, 5 sites da Internet especialmente escolhidos pelo autor e integração com um sistema de tira-dúvidas utilizandose o correio eletrônico. Contém ainda duas poderosas ferramentas de recuperação de dados (índice e localizar), além da possibilidade de impressão de páginas selecionadas. A finalização, edição e duplicação só foram possíveis graças ao importante apoio do programa BANESPA Universidades.

O Centro Virtual de Emergências Veterinárias (http://www.cevap.org.br), recentemente disponibilizado na Internet, vem acrescentar informações técnicas de extrema importância, que certamente con- tribuirão com o ensino à distância da Medicina Veterinária. Estão à disposição do usuário dezenas de imagens de animais peçonhentos (serpentes e anfíbios), facilitando assim sua identificação, abordagem clínica, tratamento e profilaxia dos acidentes. Além disso, encontram-se à disposição textos possíveis de serem impressos onde o profissional terá a possibilidade de consultar e indicar o tratamento mais adequado para cada caso. Deve ser salientado que essas páginas encontram-se em permanente evolução, e que brevemente estarão disponíveis os Acidentes com Plantas Tóxicas e, futuramente, os Acidentes com Artrópodes Peçonhentos.

Por fim, a construção dessas propostas irá contribuir com o ensino à distância na Medicina Veterinária, em especial como aqueles profissionais residentes no interior do país. Estes serão beneficiados sobremaneira e terão as mesmas oportunidades daqueles residentes nos grandes centros universitários.

\section{SUMMARY}

The authors present a brief report on the importance of publications about toxinology in veterinary medicine. These publications contribute to a high standard teaching of veterinary medicine in Brazil as well as provide a constant updating for professionals in this field. The context and importance of toxinology are also briefly described as well as printed material and CD-ROM provided by CEVAP (Center for Studies on Venoms and Venomous Animals). In the Internet, the Virtual Toxinology Center has several pages on Veterinary Emergencies.

Key words: toxinology, toxicology, toxins, venomous animals.

\section{REFERÊNCIAS BIBLIOGRÁFICAS}

1 - BARRAVIERA, B. Editoração eletrônica científica: apostando em uma nova mídia. Botucatu: FUNDIBIO, 1997, p.222.

2 - BARRAVIERA, B. O ensino dos acidentes por animais peçonhentos nas Escolas Médicas brasileiras. Rev. Soc. Bras. Med. Trop. v. 25, p.203-4, 1992.

3 - BARRAVIERA, B. Estudo clínico dos acidentes ofídicos, Botucatu: EPUB, 1999. (CD-ROM).
4 - BARRAVIERA, B. A importância do ensino da toxinologia. Rev. UNIMED, v. 8, p.60-1, 1996.

5 - BARRAVIERA, B. Revolution in scientific publication. J. Venom. Anim. Toxins, v.2, p.77-8, 1996a.

6 - BARRAVIERA, B. Venenos: aspectos clínicos e terapêuticos dos acidentes com animais peçonhentos. Botucatu: EPUB, 1998, $412 \mathrm{p}$. 